\title{
FAITHFUL UNIFORMLY CONTINUOUS REPRESENTATIONS OF LIE GROUPS
}

\author{
DENIS LUMINET AND ALAIN VALETTE
}

\section{Introduction}

Which connected real Lie groups embed continuously into the invertible group of some unital Banach algebra? This paper gives the (fairly simple) answer: only the linear groups, that is, those embedding continuously into $\mathrm{GL}_{n}(\mathbb{C})$ for $n$ big enough.

The point is that, although any connected real Lie group $G$ is locally isomorphic to some linear group (a corollary to Ado's theorem), linearity need not be preserved under coverings: in fact, no non-trivial covering group of $\mathrm{SL}_{2}(\mathbb{P})$ is linear (see [16, Chapter 2, Exercise 15(b)], or Example 1 below); on the other hand, there is a nonlinear group covered by the 3-dimensional Heisenberg group (see Example 2). It is interesting to notice that, if $G$ is linear, there exists for $n$ big enough a continuous faithful representation $G \rightarrow \mathrm{GL}_{n}(\mathbb{C})$ with closed range [4, Theorem 9].

A representation of the topological group $G$ on a complex Banach space $E$ is said to be uniformly continuous if the homomorphism $G \rightarrow \mathrm{GL}(E)$ is continuous when $\mathrm{GL}(E)$ is endowed with the norm topology. Uniformly continuous representations of real Lie groups are rather special representations since, via the differential, the Lie algebra acts by bounded operators. Singer [15] (for unitary representations) and Gurarie [5] (in general) showed that a connected real Lie group $G$ admits a faithful, uniformly continuous, uniformly bounded representation if and only if $G$ is the direct product of a compact Lie group and a real vector space; this in turn is equivalent to the fact that $G$ embeds continuously into $U(n)$ for $n$ big enough. Our Theorem A can be seen as an analogue of these results when one drops the assumption of uniform boundedness.

THEOREM A. Let $G$ be a connected real Lie group. The following properties are equivalent:

(i) $G$ is linear;

(ii) the continuous algebra seminorms separate the points of $\mathscr{E}^{\prime}(G)$;

(iii) the uniformly continuous representations separate the points of $G$;

(iv) $G$ admits a faithful, uniformly continuous representation;

(v) $G$ embeds continuously into the invertible group of a unital Banach algebra.

Recall that $\mathscr{E}^{\prime}(G)$ denotes the convolution algebra of compactly supported distributions on $G$. Endowed with the strong dual topology, it becomes an algebra with jointly continuous multiplication (see [14, Théorème VI.IV]). Theorem A says

Received 20 February 1992; revised 31 July 1992.

1991 Mathematics Subject Classification 22E15.

J. London Math. Soc. (2) 49 (1994) 100-108 
that $\mathscr{E}^{\prime}(G)$ has enough continuous algebra seminorms if and only if $G$ is linear. The criterion for $\mathscr{E}^{\prime}(G)$ to admit a continuous algebra norm is given by the following theorem.

THEOREM B. Let $G$ be a connected real Lie group. Then $\mathscr{E}^{\prime}(G)$ embeds continuously into a Banach algebra if and only if $G$ is nilpotent and simply connected.

Theorems A and B are our main results, and their proof occupies most of the paper. Theorem A will be proved in the following way: in Section 2, the implications (i) $\Rightarrow$ (ii) and (ii) $\Rightarrow$ (iii) will be shown in Propositions 1 and 2 respectively; the proof of the most difficult implication (iii) $\Rightarrow$ (i) occupies Section 3. Observe further than (i) $\Rightarrow$ (v) and (iv) $\Rightarrow$ (iii) are obvious. Concerning (v) $\Rightarrow$ (iv), if $G$ embeds continuously into the invertible group $A^{-1}$ of the Banach algebra $A$, then by letting $A$ act on itself by the left regular representation one obtains a faithful uniformly continuous representation of $G$.

Section 4 will be devoted to the proof of Theorem B; it will rely on the implication (i) $\Rightarrow$ (ii) from Theorem $A$.

The Appendix has a somewhat different flavour. Our Theorem A illustrates the dramatic difference between strongly continuous and uniformly continuous representations; however, we show thàt on certain infinite-dimensional Banach spaces, such as $\ell^{\infty}$ and $L^{\infty}$, any strongly continuous representation of a connected real Lie group is automatically uniformly continuous.

Throughout this paper, $G$ will denote a connected real Lie group, and $e$ its neutral element.

\section{Proof of Theorem A (start)}

Lemma 1. Assume that $G$ is linear; let $\mathscr{C}$ be the algebra (under pointwise multiplication) of all matrix coefficients of continuous finite-dimensional representations of $G$. Then $\mathscr{C}$ is dense in $C^{\infty}(G)=\mathscr{E}(G)$.

Proof. First note that $\mathscr{C}$ is stable under conjugation (because of conjugate representations) and that $\mathscr{C}$ separates points of $G$ (because $G$ is linear). The classical Stone-Weierstrass Theorem shows that $\mathscr{C}$ is dense in the space $C(G)$ of continuouis functions on $G$. To prove that $\mathscr{C}$ is dense in $C^{\infty}(G)$, we appeal to Nachbin's exteinsion of the Stone-Weierstrass Theorem for smooth functions [13]. The extra assumption we need to check is: for every $g \in G$ and non-zero tangent vector $Y$ at $g$, there exists a function $f \in \mathscr{C}$ such that $Y f(g) \neq 0$. Now write

$$
Y f(g)=\left.\frac{d}{d t} f(\operatorname{Exp} t X \cdot g)\right|_{t=0},
$$

where $X$ belongs to the Lie algebra of $G$. Let $\alpha$ be a faithful continuouis rèpresentation $G \rightarrow \mathrm{GL}_{n}(\mathbb{C})$; denote by $\alpha_{*}$ the differential of $\alpha$, and by $\phi_{\xi, \eta}$ the matrix coefficient of $\alpha$ associated with $\xi \in \mathbb{C}^{n}$ and $\eta \in\left(\mathbb{C}^{n}\right)^{\prime}$; then $Y \phi_{\xi, \eta}(g)=\phi_{\xi,\left(\alpha_{*}(x)\right)^{l} \eta}(g)$ will be non-zero for some $\xi, \eta$.

We thank Henrik Stetkaer for pointing out Nachbin's paper to us.

An easy consequence is the implication (i) $\Rightarrow$ (ii) of Theorem $\mathrm{A}$. 
Proposition 1. Assume that $G$ is linear. Then, for any non-zero $T \in \mathscr{E}^{\prime}(G)$, there exists a continuous algebra seminorm $v$ on $\mathscr{E}^{\prime}(G)$ such that $v(T) \neq 0$.

Proof. By Lemma 1, we find a coefficient $\phi_{\xi, \eta}$ of some continuous representation $\alpha: G \rightarrow \mathrm{GL}_{n}(\mathbb{C})$ such that $T\left(\phi_{\xi, \eta}\right) \neq 0$. Then the map

$$
v: \mathscr{E}^{\prime}(G) \rightarrow \mathbb{R}^{+} \text {given by } S \rightarrow\|\alpha(S)\|
$$

( $\|\cdot\|$ being any algebra norm on the $n \times n$ matrices) will define a continuous algebra seminorm on $\mathscr{E}^{\prime}(G)$, with $v(T) \neq 0$.

We now show the implication (ii) $\Rightarrow$ (iii) of Theorem $\mathrm{A}$.

Proposition 2. Assume that continuous algebra seminorms separate points of $\mathscr{E}^{\prime}(G)$. Then uniformly continuous representations separate points of $G$.

Proof. First notice that $G$ embeds continuously into the invertible group of $\mathscr{E}^{\prime}(G)$, by mapping $g$ to $\delta_{g}$ (continuity follows from the Mean Value Theorem applied to the first order derivatives of functions in $\mathscr{E}(G)$ ). For $g \in G, g \neq e$, we can find a continuous algebra seminorm $v$ on $\mathscr{E}^{\prime}(G)$ such that $v\left(\delta_{g}-\delta_{e}\right) \neq 0$; by separating/ completing, we get a unital Banach algebra $A_{v}$ with a continuous homomorphism $\beta_{v}: G \rightarrow A_{v}^{-1}$ such that $\beta_{v}(g) \neq 1$; finally, passing to the left regular representation of $A_{v}$, as in the last part of Section 1, we obtain a uniformly continuous representation $\alpha_{v}: G \rightarrow \mathrm{GL}\left(A_{v}\right)$ such that $\alpha_{v}(g) \neq \mathrm{Id}$.

REMARK. Let $M_{c}(G)$ be the convolution algebra of compactly supported Radon measures on $G$, endowed with the strong dual topology. Then the embedding $G \rightarrow$ $M_{c}(G)$ given by $g \rightarrow \delta_{g}$ is not continuous (except for $G=\{e\}$ ). Notice in passing that $M_{c}(G)$ always has a continuous algebra norm, given by the total variation of a measure. This means that there is no analogue of Theorems A and B with $\mathscr{E}^{\prime}(G)$ replaced by $M_{c}(G)$.

\section{Proof of Theorem A (conclusion)}

Let $G=R S$ be a Levi-Malcev decomposition of $G$, with $R$ the solvable radical and $S$ a maximal semisimple subgroup of $G$.

Proposition 3. The group $G$ is linear if and only if $R$ and $S$ are.

Proof. See [10, XVIII.4.2].

Therefore, it suffices to prove the implication (iii) $\Rightarrow$ (i) of Theorem A for $G$ semisimple (see Proposition 4) and for $G$ solvable (see Proposition 5).

Proposition 4. Assume that $G$ is semisimple. If the uniformly continuous representations separate points of $G$, then $G$ is linear.

Proof. We shall denote by $\pi: \tilde{G} \rightarrow G$ the universal covering homomorphism, and by $G^{*}$ the range of the complexification $\sigma: \tilde{G} \rightarrow \tilde{G}_{\mathbb{C}}$. 
We claim that every uniformly continuous representation $\alpha: \tilde{G} \rightarrow \operatorname{GL}(E)$ factors through $G^{*}$. Indeed, the complexified differential $\alpha_{*}$ provides a representation of the complex Lie algebra $g_{\mathbb{C}}$ by bounded operators, which we integrate to a uniformly continuous representation of $\tilde{G}_{\mathfrak{C}}$, and finally restrict to $G^{*}$. This proves the claim.

Thus, if $G$ admits enough uniformly continuous representations, we have $\operatorname{Ker} \sigma \subseteq \operatorname{Ker} \pi$, so that $G$ is a quotient of $G^{*}$; but any such quotient is linear, by [10, XVII.3.3].

EXAMPLE 1. Let $G$ be a non-trivial covering group of $\mathrm{SL}_{2}(\mathbb{R})$; then $\widetilde{G}_{\mathbb{C}}=\mathrm{SL}_{2}(\mathbb{C})$ and $G^{*}=\mathrm{SL}_{2}(\mathbb{R})$. The claim in the proof of Proposition 4 shows that any uniformly continuous representation of $G$ factors through $G^{*}$. In particular, so does any finitedimensional continuous representation of $G$, meaning that $G$ is not linear; the proof of this fact, sketched in [16, Chapter 2, Exercise 15(b)], rests on a similar idea.

We now address the difficult case, that is, the solvable case. Before proving Proposition 5, we need some preliminary results.

LEMMA 2. Assume that $G$ is solvable. The following properties are equivalent:

(a) $G$ is linear;

(b) the commutator subgroup $G^{\prime}$ is closed in $G$, and does not contain any non-trivial compact subgroup;

(c) the closure $\mathrm{cl} G^{\prime}$ of $G^{\prime}$ does not contain any non-trivial compact subgroup.

Proof. The equivalence $(\mathrm{a}) \Leftrightarrow(\mathrm{b})$ is shown in [10, XVIII.3.2]. The implication (b) $\Rightarrow$ (c) is clear. To prove the converse, let $K$ be any compact subgroup of $G$. Then $K \cap \mathrm{cl} G^{\prime}=\{e\}$, so $K \cap G^{\prime}=\{e\}$; in particular, $K \cap G^{\prime}$ is closed in $K$. It follows from [10, Chapter XVI, Exercise 2] that $G^{\prime}$ is closed in $G$.

Other conditions equivalent to linearity of a solvable $G$ are given in [4, Theorem 5]. We present now a couple of examples; the first one was promised in Section 1.

EXAmple 2. Denote by $H$ the 3-dimensional real Heisenberg group, and let $\Gamma$ be a non-trivial discrete subgroup of the centre. Then $G=H / \Gamma$ is not linear, since $G^{\prime}=Z(G)$ is a 1-dimensional torus (see also [10, Chapter XVIII, Exercise 1].

EXAmPle 3 [10, Chapter XII, Exercise 3]. With $H$ as above, let $\tilde{G}=H \times \mathbb{R}$; the centre $Z(\tilde{G})$ is a 2-dimensional vector space in which the commutator subgroup $\tilde{G}^{\prime}$ appears as a 1-dimensional subspace. Let $\Gamma$ be a lattice in $Z(\tilde{G})$, and define $G=\tilde{G} / \Gamma$. Either $\tilde{G}^{\prime} \cap \Gamma=\{e\}$, in which case $G^{\prime}$ is a real line densely embedded into a 2 dimensional torus, or $\widetilde{G}^{\prime} \cap \Gamma \neq\{e\}$, and then $G^{\prime}$ turns out to be a 1-dimensional torus (this situation being analogous to Example 2). In both cases, Lemma 2 shows that $G$ is not linear.

Lemma 3. Assume that $G$ is solvable. Let $K$ be a compact subgroup of $\mathrm{cl} G^{\prime}$. Then $K$ is central in $G$. 
Proof. Recall that $G^{\prime}$ is a nilpotent subgroup of $G$ (see [10, XI.1.3]); so $\mathrm{cl} G^{\prime}$ is nilpotent as well [1, Chapter V, Proposition 10a, Corollary]. Now Goto proved [4, Lemma 13] that a compact subgroup in a normal nilpotent Lie subgroup of $G$ is central in $G$.

We shall need the following definition, inspired by [8, Chapter 1 , Definition 1].

Definition 1. Let $\alpha$ be a strongly continuous representation of a locally compact group $H$ on a Banach space $E$. We say that $\alpha$ almost has invariant vectors if, for every $\varepsilon>0$ and every compact subset $K$ of $H$, there exists a vector $\xi$ of norm 1 in $E$ such that

$$
\|\alpha(g) \xi-\xi\|<\varepsilon \quad \text { for any } g \in K
$$

LEMMA 4. Let $K$ be a compact group. If the strongly continuous representation $\alpha: K \rightarrow \mathrm{GL}(E)$ almost has invariant vectors, then $\alpha$ has non-zero invariant vectors.

Proof (Compare with [8, Chapter 1, Example 5]). Let $d k$ be the normalized Haar measure on $K$. Let $\xi \in E$ be a unit vector such that

Define

$$
s=\sup \{\|\alpha(k) \xi-\xi\|: k \in K\}<\frac{1}{2} .
$$

$$
\eta=\int_{K} \alpha(k) d k
$$

The vector $\eta \in E$ is clearly invariant; we still need to show that $\eta \neq 0$. Let $f \in E^{\prime}$ be such that $\|f\|=1$ and $f(\xi)>\frac{1}{2}$. Then

$$
|f(\xi)-f(\eta)|=\left|\int_{K} f(\alpha(k) \xi-\xi) d k\right| \leqslant s<\frac{1}{2},
$$

so that $f(\eta) \neq 0$ and $\eta \neq 0$.

Lemma 5. Let $G$ be a connected solvable real Lie group, and let $\alpha: G \rightarrow \operatorname{GL}(E)$ be a uniformly continuous representation on the non-zero Banach space $E$. Then the restriction $\left.\alpha\right|_{\mathrm{cl} G^{\prime}}$ almost has invariant vectors.

Proof. We denote by $\mathfrak{g}$ (respectively $\mathfrak{h}$ ) the Lie algebra of $G$ (respectively $\operatorname{cl} G^{\prime}$ ). We appeal to the following result of Gurarie and Lyubich [7] (it may be seen as an infinite-dimensional version of Lie's theorem): there exists a continuous character $\chi: G \rightarrow \mathbb{C}^{-1}$ and a sequence $\left(\xi_{m}\right)_{m \in \mathbb{N}}$ of unit vectors in $E$ such that, for any $X \in \mathfrak{g}$ :

$$
\lim _{m \rightarrow \infty}\left\|\alpha_{*}(X) \xi_{m}-\chi_{*}(X) \xi_{m}\right\|=0
$$

(where $\alpha_{*}, \chi_{*}$ denote the differentials of $\alpha, \chi$ ). Since $\chi$ is trivial on $\mathrm{cl} G^{\prime}$, this implies that

$$
\lim _{m \rightarrow \infty}\left\|\alpha_{*}(X) \xi_{m}\right\|=0 \quad \text { for } X \in \mathfrak{h} .
$$

Let $X_{1}, \ldots, X_{n}$ be a basis of $\mathfrak{h}$, and $C$ be a compact subset of $\mathrm{cl} G^{\prime}$. Since $\mathrm{cl}^{\prime}$ is nilpotent, it is exponential, and we can find an $a>0$ such that any $g \in C$ can be written 
as $g=\operatorname{Exp}_{G} \sum_{i=1}^{n} t_{i} X_{i}$, with $\left|t_{i}\right|<a$ for $i=1, \ldots, n$. Let us write $\sum_{i=1}^{n} t_{i} X_{i}=X$ and $b=\sum_{i=1}^{n}\left\|\alpha_{*}\left(X_{i}\right)\right\|$. Then

$$
\begin{aligned}
\left\|\alpha(g) \xi_{m}-\xi_{m}\right\| & =\left\|\sum_{k=1}^{\infty} \frac{\alpha_{*}(X)^{k}}{k !} \xi_{m}\right\| \\
& \leqslant \sum_{k=1}^{\infty} \frac{\left\|\alpha_{*}(X)\right\|^{k-1}}{k !}\left\|\alpha_{*}(X) \xi_{m}\right\| \\
& \leqslant \sum_{k=1}^{\infty} \frac{(a b)^{k-1}}{k !} a \sum_{i=1}^{n}\left\|\alpha_{*}\left(X_{i}\right) \xi_{m}\right\| \\
& =\frac{1}{b}\left(e^{a b}-1\right) \sum_{i=1}^{n}\left\|\alpha_{*}\left(X_{i}\right) \xi_{m}\right\|,
\end{aligned}
$$

which goes to 0 uniformly on $C$ as $m \rightarrow \infty$.

LeMma 6. Assume that $G$ is solvable. Let $K$ be a compact subgroup of $\mathrm{cl} G^{\prime}$. Then $K$ is contained in the kernel of every uniformly continuous representation of $G$.

Proof. Let $\alpha: G \rightarrow \mathrm{GL}(E)$ be a uniformly continuous representation. Then $E$ splits as a direct sum to $\oplus_{\chi \in \hat{K}} E_{\chi}$, the action of $K$ on each $E_{\chi}$ being isotypic and homothetic (see for example [9, V.3.1]; it follows from [6, Remark 1] that this direct sum is actually finite, although we shall not use this fact). By Lemma $3, K$ is central in $G$; so each component $E_{\chi}$ is $\alpha(G)$-invariant. Now, the combination of Lemmas 4 and 5 shows that, provided $E_{\chi}$ is non-zero, the restriction of $\alpha$ to $E_{\chi}$ must have nonzero $\alpha(K)$-invariant vectors. This means that only the trivial character of $K$ appears in the direct sum.

We can now finish the proof of Theorem A.

Proposition 5. Assume that $G$ is solvable. If the uniformly continuous representations separate points of $G$, then $G$ is linear.

Proof. By Lemma 6, cl $G^{\prime}$ does not contain any non-trivial compact subgroup. The result now follows from Lemma 2.

Before leaving Theorem A, we remark that it has as a consequence the following result of Goto ([4], Theorem 8): a connected real Lie group is linear if and only if it has a separating family of continuous finite-dimensional representations.

\section{Proof of Theorem B}

Let $G$ be a connected real Lie group, $g$ its complexified Lie algebra, and $\mathscr{U}(\mathfrak{g})$ the enveloping algebra of $g$. The latter may, and will, be identified with the subalgebra of $\mathscr{E}^{\prime}(G)$ consisting of all distributions supported within $\{e\}$.

Theorem B states that, in order for $\mathscr{E}^{\prime}(G)$ to admit a continuous algebra norm, it is necessary and sufficient for $G$ to be nilpotent and simply connected. The necessity will follow from Lemmas 7 and 8.

LEMMA 7. The algebra $\mathscr{U}(\mathrm{g})$ admits an algebra norm if and only if $\mathrm{g}$ is nilpotent. 
Proof. See [12, main result].

Therefore, if $G$ is not nilpotent, then $\mathscr{U}(\mathfrak{g})$ is not normable, and there is no (continuous or not) algebra norm on $\mathscr{E}^{\prime}(G)$.

Lemma 8. Let $\mathbb{T}$ be the 1-dimensional torus. There is no algebra norm on $\mathscr{E}^{\prime}(\mathbb{T})$.

Proof. Assume by contradiction that $\|\cdot\|$ is such a norm. Denote by $\delta^{\prime}$ the derivative of the Dirac measure $\delta$ at the neutral element. We can find $n \in \mathbb{N}$ such that $n>\left\|\delta^{\prime}\right\|$. Then $\delta^{\prime}-$ in $\delta$ would be invertible in the $\|\cdot\|$-completion of $\mathscr{E}^{\prime}(\mathbb{T})$; but if $f(\theta)=e^{i n \theta}$, then

$$
\left(\delta^{\prime}-i n \delta\right) * f=0
$$

showing that $\delta^{\prime}-i n \delta$ is a divisor of zero, a contradiction.

Now, if $G$ is not simply connected, it contains a closed subgroup isomorphic to $\mathbb{T}$ (see [10, XVI.1.2]); so $\mathscr{E}^{\prime}(\mathbb{T})$ embeds continuously into $\mathscr{E}^{\prime}(G)$. Again we conclude that $\mathscr{E}^{\prime}(G)$, containing a non-normable subalgebra, does not admit any algebra norm.

The converse part of Theorem B is slightly more difficult. We shall prove it first for groups of triangular matrices (see Proposition 6), and then show how to derive the conclusion for arbitrary simply connected nilpotent groups.

Fix $n \geqslant 2$, and let

$$
\begin{aligned}
& R=\left\{\left(a_{i j}\right) \in \mathrm{GL}_{n}(\mathbb{R}): a_{i j}=0 \text { if } i>j, \text { and } a_{i i}>0 \text { for all } i\right\}, \\
& N=\left\{\left(a_{i j}\right) \in R: a_{i i}=1 \text { for all } i\right\} .
\end{aligned}
$$

Proposition 6. The topological algebra $\mathscr{E}^{\prime}(N)$ is normable.

Proof. Consider, for $t>0$, the diagonal matrix in $R$ :

$$
a_{t}=\operatorname{diag}\left(t, t^{2}, \ldots, t^{n}\right) \text {. }
$$

Let $\left(e_{i j}\right)_{i<j}$ be the canonical basis of the Lie algebra $n$ of $N$. For $t>0$, we have

$$
\text { Ad } a_{t}\left(e_{i j}\right)=t^{i-j} e_{i j} \text {. }
$$

Since $R$ is linear, Proposition 1 and its proof assert that there exists a separating family $\mathscr{F}$ of continuous finite-dimensional representations of $\mathscr{E}^{\prime}(R)$. Fix $\alpha: \mathscr{E}^{\prime}(R) \rightarrow$ $\mathrm{GL}\left(E_{\alpha}\right)$ belonging to $\mathscr{F}$. For $t$ (depending on $\alpha$ ) large enough, we have

$$
\left\|\alpha \circ \operatorname{Ad} a_{t}\left(e_{i j}\right)\right\| \leqslant 1 \text { for } i<j .
$$

Since $\alpha \circ \operatorname{Ad} a_{t}$ and $\alpha$ are equivalent representations, we may as well assume that $\left\|\alpha\left(e_{i j}\right)\right\| \leqslant 1$ for $i<j$ and for any $\alpha \in \mathscr{F}$. So

$$
\sup _{\alpha \in \mathscr{F}}\|\alpha(X)\|<\infty \quad \text { for any } X \in \mathfrak{n} \text {. }
$$

Now we can construct, as in [12], a representation of $n$ by bounded operators, acting on the $\ell^{1}$-direct sum $E$ of the $E_{\alpha}$ : we define

$$
\beta=\left.\underset{\alpha \in \mathscr{F}}{\bigoplus} \alpha\right|_{n}
$$

Then $\beta: \mathrm{n} \rightarrow \mathscr{L}(E)$ is a Lie algebra homomorphism. Since $N$ is simply connected, $\beta$ integrates to a uniformly continuous representation $N \rightarrow \mathrm{GL}(E)$, which finally yields $\gamma: \mathscr{E}^{\prime}(N) \rightarrow \mathscr{L}(E)$, a continuous algebra homomorphism, with

$$
\operatorname{Ker} \gamma=\bigcap_{\alpha \in \mathscr{F}} \operatorname{Ker}\left(\left.\alpha\right|_{\mathscr{E}^{\prime}(N)}\right)=\{0\}
$$


LEMma 9. Assume that $G$ is simply connected and nilpotent. Then, for $n$ large enough, there exists a continuous embedding $G \rightarrow \mathrm{GL}_{n}(\mathbb{R})$, whose range is contained in $N$.

Proof. See [10, XVIII.3.1]. Observe that the range of an embedding $G \rightarrow N$ is always closed because Exp: $\mathfrak{n} \rightarrow N$ is a diffeomorphism.

Theorem B now becomes clear: the embedding $G \rightarrow N$ induces an embedding $\mathscr{E}^{\prime}(G) \rightarrow \mathscr{E}^{\prime}(N)$, and $\mathscr{E}^{\prime}(N)$ is normable by Proposition 6.

EXAMPLE 4. When $G$ is nilpotent and simply connected, it is not true that every algebra norm on $\mathscr{U}(\mathrm{g})$ extends to an algebra norm on $\mathscr{E}^{\prime}(G)$; in other words, a monomorphism $\mathscr{U}(\mathrm{g}) \rightarrow \mathscr{L}(E)$ always yields a homomorphism $\mathscr{E}^{\prime}(G) \rightarrow \mathscr{L}(E)$, but the latter need not be injective.

To see this, consider $G=\mathbb{R}^{2}$, a simply connected additive real Lie group, on which we use $t_{1}, t_{2}$ as coordinates. Passing to the complex Fourier transform, we identify $\mathscr{U}(\mathrm{g})$ with $\mathbb{C}\left[z_{1}, z_{2}\right]$, and $\mathscr{E}^{\prime}(G)$ appears as an algebra of entire functions of $z_{1}, z_{2}$ (Paley-Wiener class). Define

$$
K=\left\{\left(z_{1}, z_{2}\right) \in \mathbb{C}^{2}: e^{-i z_{2}}=z_{1} \text { with }\left|z_{1}\right| \leqslant 1,\left|z_{2}\right| \leqslant 1\right\} .
$$

The supremum on $K$ defines an algebra norm on $\mathbb{C}\left[z_{1}, z_{2}\right]$. Consider now the distribution

$$
T=\delta_{(0,1)}+i \frac{\partial \delta_{(0,0)}}{\partial t_{1}}
$$

with support $\{(0,0),(0,1)\}$ (so that $T$ does not belong to $\mathscr{U}(\mathrm{g})$ ); then $\hat{T}\left(z_{1}, z_{2}\right)=$ $e^{-i z_{2}}-z_{1}$ vanishes on $K$. Therefore, the supremum on $K$ will yield only a seminorm on $\mathscr{E}^{\prime}(G)$.

This example shows that Proposition 1 is an essential ingredient in the proof of Proposition 6.

\section{Appendix}

We prove here that there are infinite-dimensional Banach spaces on which any strongly continuous representation of a connected real Lie group is uniformly continuous.

Definition 2. A Banach space $E$ has the Dunford-Pettis property if, for any sequence $\left(x_{n}\right)_{n \in \mathbb{N}}$ in $E$ converging weakly to $x$, and any sequence $\left(f_{n}\right)_{n \in \mathbb{N}}$ in $E^{\prime}$ converging weakly to $f$, one has

$$
\lim _{n} f_{n}\left(x_{n}\right)=f(x)
$$

Definition 3. A Banach space $E$ has the Grothendieck property if every weak* convergent sequence in $E^{\prime}$ also converges in the weak topology on $E^{\prime}$.

If $K$ is a compact space, it is known that $C(K)$ always has the Dunford-Pettis property, and that it has the Grothendieck property when $K$ is stonian, that is, the closure of an open set is open; see for example [11], and the references therein. In particular $L^{\infty}$ and $\ell^{\infty}$ have both the Dunford-Pettis and the Grothendieck properties. 
Proposition 7. Let E be a Banach space having both the Dunford-Pettis and the Grothendieck properties. Any strongly continuous representation $\alpha$ of a connected real Lie group $G$ on $E$ is uniformly continuous.

Proof. We appeal to a result of Lotz ([11, Theorem 3]; see also [3] for a short proof): any strongly continuous one-parameter semigroup of operators on $E$ is uniformly continuous. Thus, if $X$ is an element in the Lie algebra $g$ of $G$, the oneparameter group $\left(\alpha\left(\operatorname{Exp}_{G} t X\right)\right)_{t \in \mathbb{R}}$ is strongly continuous, hence uniformly continuous. Let then $X_{1}, \ldots, X_{n}$ be a basis of $\mathfrak{g}$; by [10, VII.4.1] there exists a number $\varepsilon>0$ and a neighbourhood $U$ of $e$ in $G$ such that any element $g \in U$ can be written uniquely as

$$
g=\operatorname{Exp}_{G} t_{1} X_{n} \ldots \operatorname{Exp}_{G} t_{n} X_{n}
$$

with $\left|t_{i}\right|<\varepsilon$ for $i=1, \ldots, n$. So the homomorphism $\alpha: G \rightarrow \operatorname{GL}(E)$ is uniformly continuous at $e$, hence everywhere.

If $\alpha$ is uniformly bounded, our Proposition 7 appears as a particular case of the Theorem in $[2, \S$ III.4].

We thank Thierry Coulhon for providing us with the references of this Appendix.

\section{References}

1. C. Chevalley, Théorie des groupes de Lie (Hermann, Paris, 1968).

2. T. Coulhon, 'Semi-groupes d'opérateurs et suites de contractions sur les espaces $L^{\infty}$ et $C(K)$ ', Thèse de 3ème Cycle, Université Pierre et Marie Curie, Paris VI, 1984.

3. T. Coulhon, 'Approximation forte et uniforme de l'identité sur les espaces $C(K)$ ', Séminaire d'Analyse Fonctionnelle, Publications Mathématiques de l'Université Paris VII 20 (1983-1984) 117-121.

4. J. Goto, 'Faithful representations of Lie groups II', Nagoya Math. J. 1 (1950) 91-107.

5. D. Gurarie, 'Banach uniformly continuous representations of Lie groups and algebras', J. Funct. Anal. 36 (1980) 401-407.

6. D. GURARIE, 'Banach uniformly continuous representations of locally compact groups', 1980, Case Western Reserve University, unpublished.

7. D. GURARIE and Y. I. LYUBICH, 'An infinite-dimensional theorem analogous to Lie's weight theorem', Functional Anal. Appl. 7 (1973) 34-36.

8. P. DE la HARPe and A. VAlette, 'La propriété (T) de Kazhdan pour les groupes localement compacts', Astérisque 175 (Soc. Math. France, Montrouge, 1989).

9. S. Helgason, Groups and geometric analysis, Pure and Applied Mathematics 113 (Academic Press, Boston, 1984).

10. G. HochsCHILD, The structure of Lie groups (Holden Day, San Francisco-London-Amsterdam, 1965).

11. H. P. LoTz, 'Uniform convergence of operators on $L^{\infty}$ and related spaces', Math. Z. 190 (1985) 207-220.

12. D. LUMinet, 'Norms on enveloping algebras', Proc. Amer. Math. Soc. 101 (1987) 65-66.

13. L. Nachbin, 'Sur les algèbres denses de fonctions différentiables sur une variété', C. R. Acad. Sci. Paris Ser. I Math. 228 (1949) 1549-1551.

14. L. Schwartz, Théorie des distributions (Hermann, Paris, 1966).

15. I. M. SingER, 'Uniformly continuous representations of Lie groups', Ann. of Math. 56 (1952) 242-247.

16. V. S. VARADARAJAN, Lie groups, Lie algebras and their representations, Graduate Texts in Mathematics 102 (Springer, New York-Berlin, 1984).

Departement Wiskunde

Vrije Universiteit Brussel

B-1050 Brussels

Belgium
Institut de Mathématiques

Université de Neuchâtel

CH-2007 Neuchâtel

Switzerland 